\title{
Effects of cholesterol on the binding of the precursor neurotransmitter tryptophan to zwitterionic membranes
}

Cite as: J. Chem. Phys. 149, 164906 (2018); https://doi.org/10.1063/1.5029430

Submitted: 14 March 2018 . Accepted: 09 October 2018 . Published Online: 25 October 2018

Huixia Lu (D), and Jordi Martí (iD)
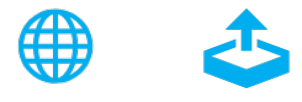

\section{ARTICLES YOU MAY BE INTERESTED IN}

Perspective: Identification of collective variables and metastable states of protein dynamics

The Journal of Chemical Physics 149, 150901 (2018); https://doi.org/10.1063/1.5049637

Absolute cross section for DNA damage induced by low-energy (10 eV) electrons:

Experimental refinements and sample characterization by AFM

The Journal of Chemical Physics 149, 164904 (2018); https://doi.org/10.1063/1.5041805

Elucidating thermal behavior, native contacts, and folding funnels of simple lattice proteins using replica exchange Wang-Landau sampling

The Journal of Chemical Physics 149, 164913 (2018); https://doi.org/10.1063/1.5026256

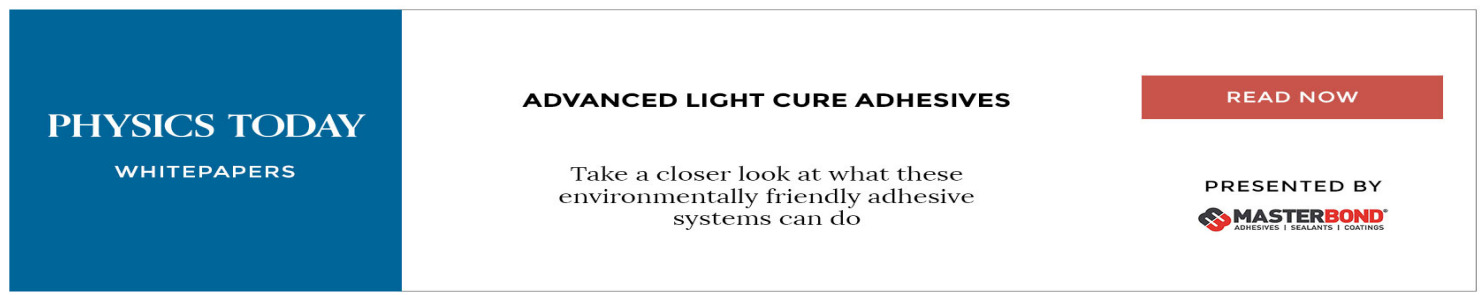




\title{
Effects of cholesterol on the binding of the precursor neurotransmitter tryptophan to zwitterionic membranes
}

\author{
Huixia $\mathrm{Lu}^{1, \mathrm{a})}$ and Jordi Martí ${ }^{2, \mathrm{~b})}$ \\ ${ }^{1}$ Department of Physics, Technical University of Catalonia-Barcelona Tech, B4-210 Northern Campus UPC, \\ 08034 Barcelona, Catalonia, Spain \\ ${ }^{2}$ Department of Physics, Technical University of Catalonia-Barcelona Tech, B5-209 Northern Campus UPC, \\ 08034 Barcelona, Catalonia, Spain
}

(Received 14 March 2018; accepted 9 October 2018; published online 25 October 2018)

\begin{abstract}
The characterization of the microscopical forces between the essential $\alpha$-amino-acid tryptophan, precursor of the neurotransmitter serotonin and of the hormone melatonin, and the basic components of cell membranes and their environments (phospholipids, cholesterol, ionic species, and water) is of central importance to elucidate their local structure and dynamics as well as the mechanisms responsible for the access of tryptophan to the interior of the cell. We have performed nanosecond molecular dynamics simulations of tryptophan embedded in model zwitterionic bilayer membranes made by di-palmitoyl-phosphatidyl-choline and cholesterol inside aqueous sodium-chloride solution in order to systematically examine tryptophan-lipid, tryptophan-cholesterol, and tryptophan-water interactions under liquid-crystalline phase conditions. Microscopic properties such as the area per lipid, lipid thickness, radial distribution functions, hydrogen-bonding lengths, atomic spectral densities, and self-diffusion coefficients have been evaluated. Our results show that the presence of tryptophan significantly affects the structure and dynamics of the membrane. Tryptophan spends long periods of time at the water-membrane interface, and it plays a central role by bridging a few lipids and cholesterol chains by means of hydrogen-bonds. The computed spectral densities, in excellent agreement with experimental infrared and Raman data, revealed the participation of each atomic site of tryptophan to the complete spectrum of the molecule. Tryptophan self-diffusion coefficients have been found to be in between $10^{-7}$ and $10^{-6} \mathrm{~cm}^{2} / \mathrm{s}$ and strongly depending of the concentration of cholesterol in the system. Published by AIP Publishing. https://doi.org/10.1063/1.5029430
\end{abstract}

\section{INTRODUCTION}

Cell membranes are limiting structures separating cell contents from external environments. They protect cellular contents, including a wide variety of organelles such as mitochondria, nucleus, lysosomes, vacuoles, Golgi apparatus, centrioles, and ribosomes surrounded by the cytoplasm, and simultaneously allow the passing of nutrients, wastes, and eventually drugs through them. Membranes only allow the movement of compounds generally with small molecular size into the cell. For such a reason, the knowledge of the mechanisms responsible for the exchange of small peptides and drugs inside the membrane is of greatest scientific interest. The principal components of human cellular membranes are phospholipids, cholesterol, and proteins, all of them embedded in ionic aqueous solution. Phospholipid membranes provide the framework to biomembranes, to which other molecules (such as proteins or cholesterol) attach. ${ }^{1}$ They consist of two leaflets of amphiphilic lipids with a hydrophilic head and one or two hydrophobic tails which self-assemble due to the hydrophobic effect. ${ }^{2}$ Since these molecules are not chemically bound

\footnotetext{
a)huixia.lu@upc.edu

b)Author to whom correspondence should be addressed: jordi.marti@upc.edu
}

between them, not only their local (vibrational) motions can be analyzed but also their long-range correlated motions (diffusion) can be measured and studied with all details with computer simulations. Cholesterol is a lipid playing a central role in maintaining the structure of the membrane and regulating their functions. ${ }^{3,4}$ It induces the membrane to adopt a liquid-ordered phase with positional disorder and high lateral mobility. ${ }^{1}$ The fluidity of the membrane is mainly regulated by the amount of cholesterol, in such a way that membranes with high cholesterol contents are stiffer than those with low amounts but keeping the appropriate fluidity for allowing normal membrane functions. There exists a big variety of experimental techniques useful to explore membrane organization and molecular interactions of small probes within the membrane, such as NMR, neutron diffraction, X-ray scattering or IR, Raman, and fluorescence spectroscopy. ${ }^{5-8}$ Among the latest techniques, fluorescence-lifetime microscopy ${ }^{9}$ can be combined with spectral information to report basic information of aspects such as metabolic profiles, photophysics, or dipolar relaxations. ${ }^{10}$

In this paper, we have focused our efforts in two directions: on the one side, the study of zwitterionic phospholipid membranes can help to understand basic biological functions of the membrane and its interaction with the environment. As an example of a prototype membrane, the one formed by 
di-palmitoyl-phosphatidyl-choline (DPPC) is, for instance, a major constituent (about $40 \%$ ) of pulmonary lungs. ${ }^{11}$ A large number of simulations and experiments have already been performed on DPPC, often including the influence of cholesterol in water environments. ${ }^{12}$ Some of these reports will allow us to test the reliability of our simulations, especially about the characterization of the physical states considered and their mechanical properties, in particular, the area per lipid of the system. On the other side, the role of proteins and drugs and their interactions with the membrane structure is undoubtedly a relevant field of research. In this work, we have considered the introduction into the lipid bilayer structure of a small biological probe which is a zwitterion itself: the aminoacid tryptophan ${ }^{6}$ (TRP), a precursor of the neurotransmitter serotonin ${ }^{13}$ which, in turn, is also the precursor of the hormone melatonin. ${ }^{14}$ Since the human body cannot synthesize tryptophan, needed to prevent diseases and death, it has to be acquired from the diet. Tryptophan is able to act as a building block in protein biosynthesis, while proteins are fundamentals required to sustain life. In addition, it helps in the regulation of human sleep. Serotonin, in turn, can be converted to melatonin (a neurohormone), which may help humans to the regulation of biological rhythms, to induce sleep, to work as a strong antioxidant, and also to contribute to the protection of the organism from carcinogenesis and neurodegenerative disorders such as Alzheimer's disease. ${ }^{15}$ Recently, some studies indicated promising antibacterial properties of tryptophan when synthesized at polyionic membranes ${ }^{16}$ and its major role in the preparation of nanoparticles able to control membrane disruption. ${ }^{17}$ So, given the importance of tryptophan, we have explored its interactions with a typical zwitterionic phospholipid membrane formed by DPPC and cholesterol in an aqueous solution of sodium chloride, analyzing its mechanical properties, the local structure, and some relevant dynamical properties such as diffusion and vibrational spectra. However, we do not state that the particular properties and data reported for tryptophan in the present work can be considered as general for a wide variety of small probes.

We provide the details of the simulations in Sec. II and explain the main results of the work in Sec. III, focusing our attention especially on the local structures of tryptophan (Sec. III A) and also on the vibrational motions of tryptophan atoms, described in Sec. III B 2. Finally, some concluding remarks are outlined in Sec. IV.

\section{METHODS}

A prototype model of a lipid bilayer membrane in a sodium chloride solution in water has been built by means of the CHARMM-GUI web-based tool. ${ }^{18,19}$ The membrane was composed by 204 lipids, distributed in two leaflets of 102 flexible DPPC $\left(\mathrm{C}_{40} \mathrm{H}_{80} \mathrm{NO}_{8} \mathrm{P}\right)$ molecules, surrounded by 4962 TIP3P $^{20}$ water (W) molecules, with 17 sodium and 17 chlorine ions, corresponding to physiological concentration, plus one zwitterionic L-tryptophan $\left(\mathrm{C}_{11} \mathrm{H}_{12} \mathrm{~N}_{2} \mathrm{O}_{2}\right)$ molecule. Eventually some of the lipids were replaced by cholesterol molecules $\left(\mathrm{C}_{27} \mathrm{H}_{46} \mathrm{O}\right)$ in such a way that two cholesterol percentages were considered: $30 \%$ and $50 \%$. Previous simulations with percentages of $10 \%, 20 \%$, and $40 \%$ showed no further relevant physical changes compared to the reported states. A benchmark state of a cholesterol-free system (labeled as $0 \%$ throughout the paper) was also considered at the temperature of $323.15 \mathrm{~K}$, in order to ensure that the simulation was performed at the liquid state since the crossover temperature for pure DPPC to become liquid has been determined to be around $314 \mathrm{~K}^{21,22}$

Sketches of the backbone structure of cholesterol, tryptophan, and DPPC are represented in Fig. 1. Each molecule was described with atomic resolution (74, 27, and 130 sites, respectively). Molecular dynamics (MD) simulations were performed with the NAMD2 simulation package ${ }^{23}$ at a fixed (human body) temperature of $310.15 \mathrm{~K}$ for the systems containing cholesterol and at the fixed pressure of $1 \mathrm{~atm}$. The reason for considering such a difference in temperature was to have the system always in liquid-crystalline phases as close as possible to the human body temperature: liquid-disordered for the cholesterol-free case and liquid-ordered (with headgroups showing liquid-like behavior, but with tailgroups showing some gel-like state, with an ordering structure larger than that of headgroups) for the setups containing 30\% and 50\% cholesterol, when the crossover temperature from the gel to liquid phase is below $310 \mathrm{~K}$ (see Ref. 21). In all cases, the temperature was controlled by a Langevin thermostat ${ }^{24}$ with a damping coefficient of $1 \mathrm{ps}^{-1}$, whereas the pressure was controlled by a Nosé-Hoover Langevin barostat ${ }^{25}$ with a damping time of $50 \mathrm{fs}$.

Considering the isobaric-isothermal ensemble, i.e., at a constant number of particles $(\mathrm{N})$, pressure $(\mathrm{P})$, and temperature (T) conditions, equilibration periods for all simulations were of more than $50 \mathrm{~ns}$. After equilibration, we recorded statistically meaningful trajectories of more than $70 \mathrm{~ns}$ in all cases through several production runs. The simulation boxes had different sizes because of the cholesterol concentrations. For instance, in the cholesterol-free system, the size was, in average, of $80 \AA \times 80 \AA \times 90 \AA$. As cholesterol concentration increased, the size of the system was systematically reduced. The simulation time step was set to $2 \mathrm{fs}$. We adopted the CHARMM $36^{26,27}$

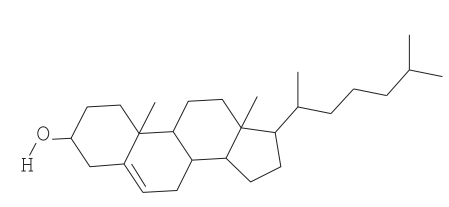

Cholesterol

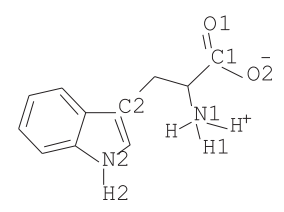

Tryptophan

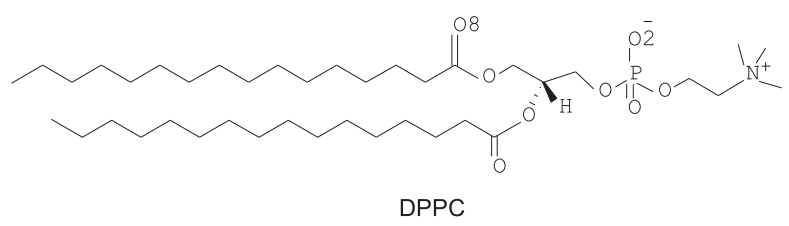

FIG. 1. Sketches of the backbone structures of DPPC, cholesterol, and tryptophan. Hydrogens bound to carbon are not shown. The highlighted sites of TRP (H1, H2, N1, N2, C1, C2, O1, and O2) and of DPPC (N, O2, and O8) will be referred in the text by the labels defined here. Due to the zwitterionic characteristics of L-tryptophan, its site $\mathrm{H} 1$ corresponds to any of the three hydrogens bound to N1, which share the positive charge. Sites O1 and O2 of TRP share the negative charge. Site C2 is close to the center of mass of the molecule. 
force field, which is able to reproduce the area per lipid in good agreement with experimental data. All bonds involving hydrogens were set to fixed lengths, allowing fluctuations of bond distances and angles for the remaining atoms. Nevertheless, during the calculation of spectral densities (see Sec. III B), all bonds including those involving hydrogens were left flexible. Van der Waals interactions were cut off at $12 \AA$ with a smooth switching function starting at $10 \AA$. Long ranged electrostatic forces were computed using the particle mesh Ewald method, ${ }^{28}$ with a grid space of about $1 \AA$ A. Such electrostatic interactions were updated every time step. Finally, periodic boundary conditions were applied in all three directions of space.

\section{RESULTS AND DISCUSSION}

A general view of the half of the system at several cholesterol concentrations is shown in Fig. 2. There, for the sake of clarity, water and DPPC have been depicted with thin sticks, whereas cholesterol, tryptophan, and the sodium and chlorine ions have been shown more explicitly. We should point out that TRP tends to stay close to the headgroup regions of the lipid and cholesterol chains during most time of the simulated trajectories: ${ }^{29}$ An estimation over the full trajectory length (cholesterol concentration of 30\%) indicated that around $70 \%$ of time TRP is attached to DPPC with the remaining time being mostly solvated by water. Furthermore, in the membrane with $30 \%$ of cholesterol, we observe that TRP at the interface is located slightly deeper than in the case with $50 \%$ of cholesterol so that a primary effect of cholesterol is a tendency to exclude TRP out of the water-membrane interface.

With the aim of ensuring that we are simulating the liquid phase of the model system as well as to efficiently characterize the order of the hydrated lipid bilayer, we computed the deuterium order parameter $S_{C D}$ which can be obtained from ${ }^{2} \mathrm{H}$ NMR experiments. This quantity was first reported by Stockton and $\mathrm{Smith}^{30}$ and later on adapted by Hofsäß et al. ${ }^{31}$ by means of an order parameter, defined for each $\mathrm{CH}_{2}$ group as follows:

$$
S_{C D}=\frac{1}{2}\left(3\left\langle\cos ^{2} \theta_{C D}\right\rangle-1\right),
$$

where $\theta_{C D}$ is the angle between the membrane normal and a $\mathrm{CH}$-bond (a CD-bond in the experiments). Brackets in Eq. (1) indicate ensemble average for all lipids. The results are shown in Fig. 3 for the two chains (sn1, sn2) of a DPPC molecule at three cholesterol concentrations. The results are in good overall agreement with both experimental ${ }^{5,32}$ and simulation studies $^{31,33}$ and confirm that the system, in the simulation conditions assumed in the present work, represents well the liquid-crystalline phase. We should note the tendency to higher ordering as cholesterol concentration increases, which is represented by profiles of $S_{C D}$ having larger maxima (around 0.2 for the cholesterol-free system versus $\sim 0.4$ for the cholesterolrich setups), a similar tendency as observed by Hofsäß et al. ${ }^{31}$ and Chiu et al. $^{34}$

One primary output from any simulation of biomembranes is the area per lipid. For a series of equilibrated MD runs at the NPT ensemble, we have monitored the surface area per
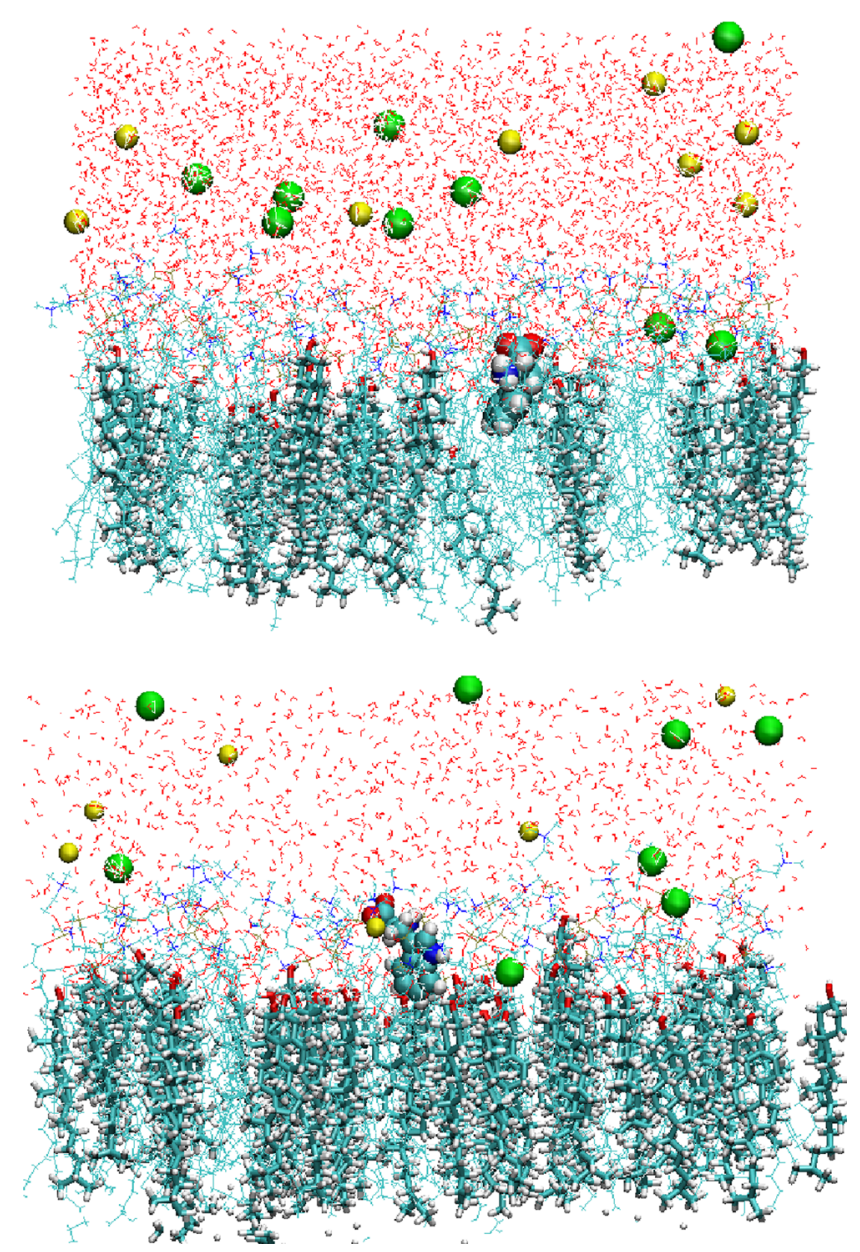

FIG. 2. Snapshots of the aqueous DPPC and cholesterol membranes with an embedded tryptophan molecule (top: 30\% of cholesterol; bottom: $50 \%$ of cholesterol). Atoms in TRP: oxygen (red), hydrogen (white), carbon (cyan), and nitrogen (blue). DPPC and water molecules are shown as thin sticks. Cholesterol is depicted as thick sticks. Sodium ions are shown in green and chlorine ions are shown in yellow.

lipid considering the total surface along the $X Y$ plane (plane parallel to the bilayer surface) divided by the number of lipids plus cholesterol in one lamellar layer. ${ }^{35}$ The area per lipid (for the final $70 \mathrm{~ns}$ of all trajectories) as a function of simulation times is reported in Fig. 4, and the averaged values are reported in Table I. The reader should note that these values for

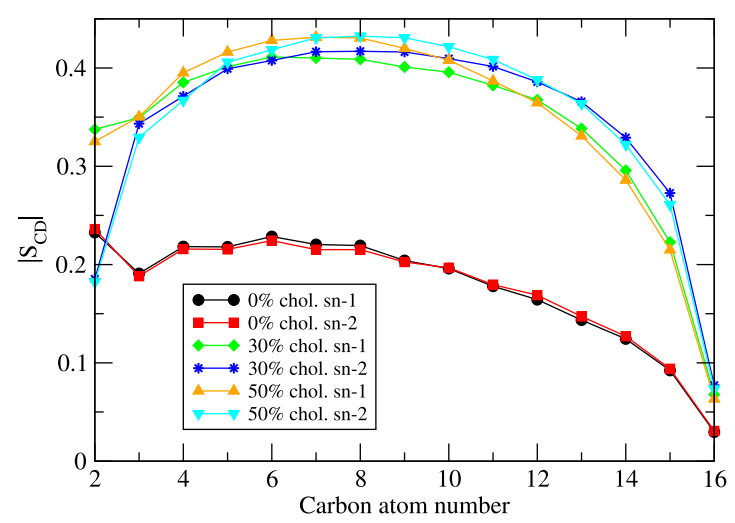

FIG. 3. Order parameter $\left|S_{C D}\right|$ for the acyl tails for three different percentages of cholesterol. 


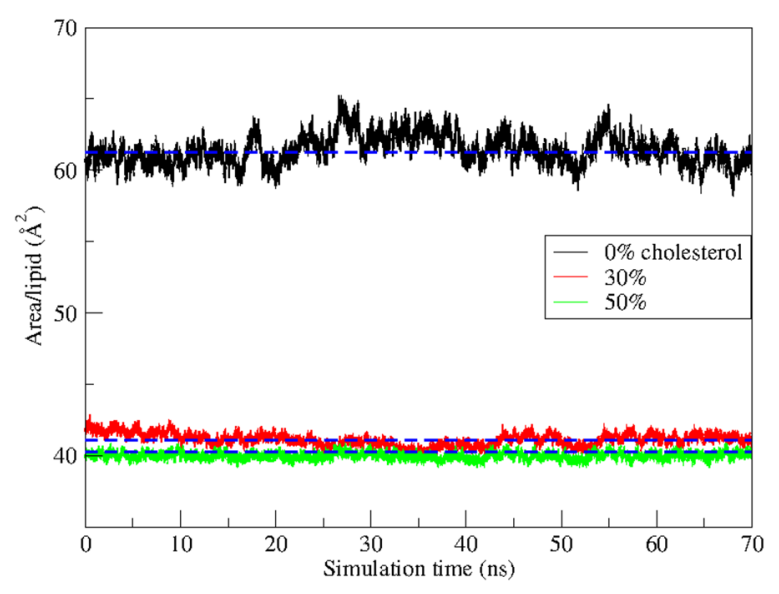

FIG. 4. Area per lipid versus cholesterol contents of the system: 0\%, 30\%, and $50 \%$. Dashed lines indicate average values reported in Table I.

the area per lipid arise naturally from the relaxation of the system at a given temperature, pressure, and number of particles and are not an imposition to fit the experimental value. The main trend observed from our data is that the areas decrease as cholesterol concentration increases, as expected. In addition, fluctuations of the area per lipid in the $0 \%$ cholesterol case are larger than those of the cholesterol-rich ensembles. We obtained an averaged value of $61.4 \AA^{2}$ for the $0 \%$ cholesterol system and smaller values around to $40 \AA^{2}$ when cholesterol is present at the membrane. These results are in overall good agreement with other computational data in a wide variety of thermodynamical conditions $5,31,34,36,37$ where the values for pure DPPC ranged between 50 and $63 \AA^{2}$ and the trend of decreasing areas for increasing cholesterol percentages is clearly reported. From the experimental side, an influential review from Nagle and Tristram-Nagle ${ }^{2}$ reported values of the area per lipid of pure DPPC membranes obtained from a wide variety of methods (NMR, X-ray, and neutron scattering) between 48 and $52 \AA^{2}$ in the gel phase $(293 \mathrm{~K})$ and between 57 and $71 \AA^{2}$ at the liquid phase $(323 \mathrm{~K})$. However, some of them were reported to be measured under wrong assumptions due to artificial undulations of the membrane sets. The best estimations were of 48 and $64 \AA^{2}$ for the gel phase and liquid phase, respectively, in the cholesterol-free case. In a quite recent study, Kučerka et al. ${ }^{22}$ found a value of $63.1 \AA^{2}$ for DPPC at $323.15 \mathrm{~K}$ by means of X-ray and neutron scattering techniques. In the present work, a sudden drop in the area per lipid is dramatically seen when cholesterol is introduced in the membrane, as expected. Edholm and Nagle $^{36}$ reported a summary of values from independent MD simulations where at the cholesterol concentration of $30 \%$, area/lipid was around $43 \AA^{2}$ and at $50 \%$, was between 37 and $39 \AA^{2}$, at $323 \mathrm{~K}$ in all

TABLE I. Mechanical properties of the membrane: area per lipid and thickness. Estimated errors are in parentheses.

\begin{tabular}{lcc}
\hline \hline Cholesterol (\%) & $A\left(\AA^{2}\right)$ & $\Delta z(\AA)$ \\
\hline 0 & $61.4(0.8)$ & $39.7(0.5)$ \\
30 & $40.8(0.2)$ & $48.9(0.4)$ \\
50 & $40.1(0.2)$ & $47.8(0.3)$ \\
\hline \hline
\end{tabular}

cases. Assuming the small difference in temperature for the cholesterol-rich systems (310 in the present work), the agreement with our results is qualitatively good. Furthermore, the results reported here are consistent with the observation that, in di-myristoyl-phosphatidyl-choline (DMPC) membranes analyzed at $303 \mathrm{~K}$, cholesterol is able to favor a phase transition from a liquid-disordered phase to a liquid-ordered phase, ${ }^{38,39}$ in particular, when cholesterol contents are higher than $30 \%$.

The variations of the thickness of the membrane may give some additional clues of the effect of cholesterol on its mechanical properties, such as its rigidity and its capability to allow the passing of species inside and outside the cell. We have obtained the thickness by computing the distance between the phosphate groups of the DPPC molecules located at the two sides of the membrane. The results are also reported in Table I.

These values of the bilayer thickness are in good overall agreement with those reported by Kučerka et al. ${ }^{22}$ These authors found a value of $39 \AA$ at $323 \mathrm{~K}$ for a cholesterol-free sample and a tendency to decrease with increasing temperatures. In the present work, we have obtained a value of $39.7 \AA$ at the cholesterol-free system and values around $48 \AA$ when cholesterol is present in the membrane. This fact is consistent with the findings of the area per lipid reported above where at higher cholesterol contents, the values have been found smaller: at large cholesterol contents, the bilayer structures are more compressed so that we have more lipid species per $\AA^{2}$ which would eventually produce the extension of the lipid tails (which are normally showing a large extent of folding for the cholesterol-free system) and the corresponding larger bilayer thickness. This is also consistent with the results shown at Fig. 3 where cholesterol-rich systems have tails significantly more ordered (gel-like) than those corresponding to the cholesterol-free case.

\section{A. Structure of the solvated tryptophan}

The local structure of a multicomponent system can be analyzed by means of normalized atomic radial distribution functions (RDFs) $g_{A B}(r)$. For a species $B$ close to a tagged species $A$, they are given by

$$
g_{A B}(r)=\frac{V\left\langle n_{B}(r)\right\rangle}{4 N_{B} \pi r^{2} \Delta r},
$$

where $n_{B}(r)$ is the number of atoms of species $B$ surrounding a given atom of species $A$ inside a spherical shell of width $\Delta r$. $V$ stands for the total volume, and $N_{B}$ is the total number of particles of species $B$. We have evaluated the local structure of the tryptophan molecule solvated by water, DPPC, and cholesterol. We will restrict ourselves to report the most relevant $g(r)$, namely, those defined for the following pairs:

1. The hydrogens "H1" of tryptophan (see Fig. 1) versus the oxygen of a water molecule ("OW"),

2. the oxygens of tryptophan ("OT," corresponding to the sites labeled "O1" and "O2;" see Fig. 1) versus the hydrogen of a water molecule ("HW"),

3. the hydrogens "H1" of tryptophan versus the oxygen of cholesterol (“OC"), 

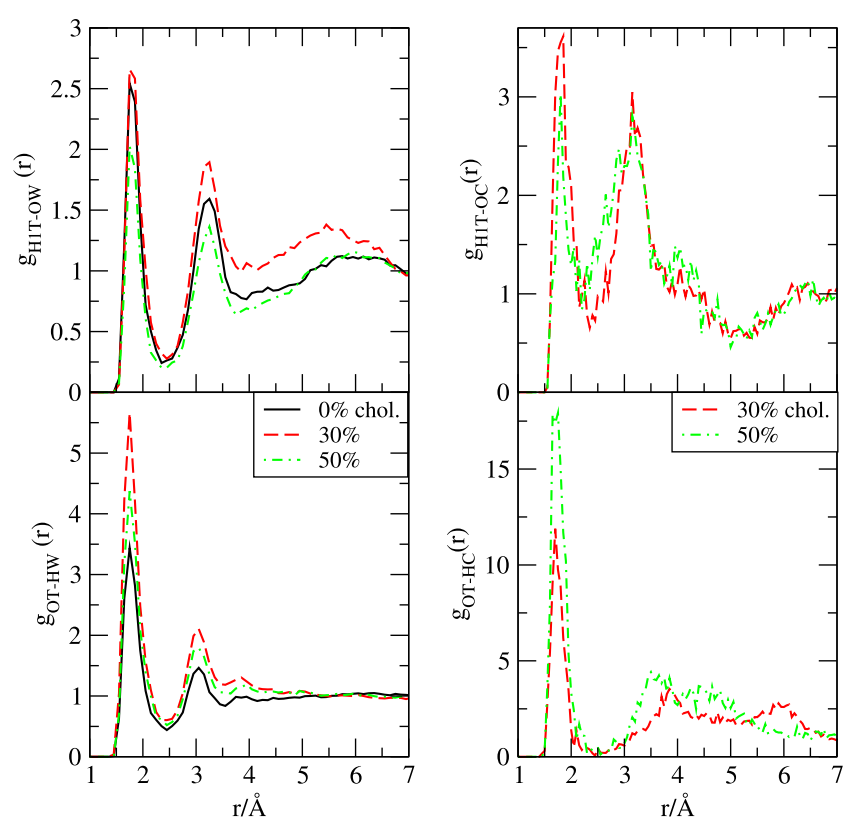

FIG. 5. Radial distribution functions for tryptophan (T) with water (W) and cholesterol (sites "O" and "H" of the hydroxyl group): H1T-OW (top left), OT-HW (bottom left), H1T-OC (top right), and OT-HC (bottom right).

4. the oxygens of tryptophan "OT" versus the hydrogen of cholesterol ("HC"), and

5. the hydrogens of tryptophan "H1" and "H2" (taken separately) versus the negatively charged oxygens of DPPC ("O2" and "O8," taken separately).

The results of the $g(r)$ defined above are presented in Figs. 5 and 6 for the three relevant tryptophan concentrations $(0 \%, 30 \%$, and $50 \%)$.

All eight radial distribution functions show some fluctuations in their profiles, the statistical noise observed in the association of TRP with cholesterol being more marked than
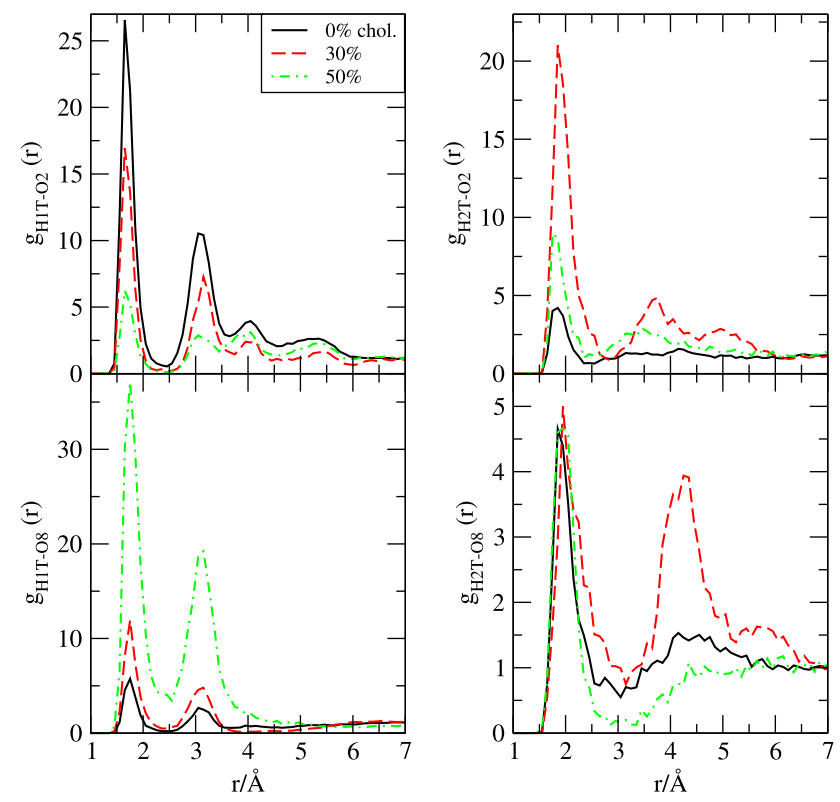

FIG. 6. Radial distribution functions for tryptophan (T) with DPPC (charged sites "H1, "H2," "O2," and "O8," see Fig. 1): H1T-O2 (top left), H1T-O8 (bottom left), H2T-O2 (top right), and H2T-O8 (bottom right). for TRP-Water and for TRP-DPPC. The fluctuations are essentially due to the fact that tryptophan is a mobile species and can be mostly solvated by DPPC or water. However, cholesterol solvation only happens during short periods of time (see below), which generates larger fluctuations of the corresponding $g(r)$ s. As a general feature, the presence of cholesterol does not affect the positions of the maxima in the $g(r)$ between TRP and water and between TRP and DPPC, although significant reduction of the heights of the maxima has been observed, especially for $g_{\mathrm{H} 1 \mathrm{~T}-\mathrm{O} 2}$. We can observe a clear first coordination shell in all cases being the binding of TRP to DPPC, the one with the highest peaks of the $g(r)$ s (see Fig. 6). In such a case, a marked second coordination shell can be also observed. The peaks located around 1.7-1.8 $\AA$ in all $g(r)$ are the signature of hydrogen bonds (HBs) established between TRP and water, TRP and DPPC, and also TRP with cholesterol. Using fluorescence spectroscopy, Liu et al. ${ }^{8}$ obtained values for the hydrogen-bond lengths of TRP and water between 1.6 and $2.1 \AA$, i.e., in the same range of the values reported in the present work.

In the present work, we have not considered the usual density profiles defined by the distance of an atomic site to the center of the lipid bilayer and instead we computed radial distribution functions. The reason is twofold: (1) we are mainly interested in the solvation structure of tryptophan instead of their distribution across the bilayer and (2) RDFs have the additional advantage to provide a direct route to the estimation of free energy barriers, ${ }^{40}$ but, since reaction coordinates for probe binding to membranes will be generally unknown and not one-dimensional but multidimensional, methods not assuming preconceived reaction coordinates such as transition path sampling ${ }^{41-43}$ or, those allowing to consider several complementary collective variables, such as metadynamics ${ }^{44,45}$ would be in order to obtain much more accurate free energy landscapes for TRP adsorption. Nevertheless, their drawback is the huge amount of computational time required in both cases, and for this reason, they are not considered in the present work.

During the long simulation runs used to collect statistically meaningful properties, we observed periods of time of about $5 \mathrm{~ns}$ where TRP was fully solvated by water, suggesting that hydration of TRP is one stable state of the system. Full TRP hydration is essentially operated through hydrogenbonding between the triplet of "H1" hydrogens of tryptophan bound to "N1" (see Fig. 1) and the water's oxygen or, equivalently, between oxygens of TRP and hydrogens in water, as shown in the left column of Fig. 5. There we can observe a sharp maximum located around $1.85 \AA$ for both $g_{\mathrm{H} 1 \mathrm{~T}-O W}$ and $g_{O T-H W}$. When cholesterol is introduced in the systems, the binding of TRP to water remains essentially unchanged, although first maxima of $g_{O T-H W}$ show larger values. First, this could be due to the exclusion of TRP off the interface operated by the presence of cholesterol and, second, because of the binding of TRP to cholesterol, as shown in the right column of Fig. 5. Interestingly, cholesterol can form hydrogen-bonds with TRP's hydrogens as indicated by the first maximum of $g_{\mathrm{H} 1 \mathrm{~T}-O C}$ and also with TRP's oxygens (shown in $g_{O T-H C}$ ). The large statistical noise in those functions might be an indication of the short life of such HB. 
Observing that TRP can establish some hydrogenbonding with cholesterol, all data we got suggest that TRP is also able to stay bound to the inner part of lipid heads during significant periods of time, in agreement with previous results indicating that in a cholesterol-free di-oleoylphosphatidyl-choline bilayer membrane, TRP tends to localize strongly in the interfacial region. ${ }^{46}$ The preferential location of TRP at the lipid-water interface was observed by de Jesus and Allen ${ }^{14}$ for membrane proteins. In the present work, we observed that the average lifetime of TRP at the interface of the DPPC bilayer is of the order of $10 \mathrm{~ns}$. Regarding TRP-DPPC binding (see Fig. 6), we have observed hydrogenbonding connections between sites "H1" and "H2" of TRP and DPPC sites "O2" and "O8" (labels according to Fig. 1), as

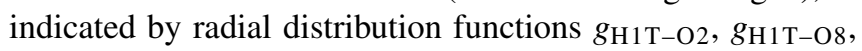
$g_{\mathrm{H} 2 \mathrm{~T}-\mathrm{O} 2}$, and $g_{\mathrm{H} 2 \mathrm{~T}-\mathrm{O} 8}$. Functions related to "H1" hydrogens in TRP (left column of Fig. 6) show a clear maximum located around $1.7 \AA$ when bound to "O2" of DPPC and around $1.75 \AA$ when bound to "O8" of DPPC. The presence of cholesterol diminishes the "H1-O2" binding, but it enhances the "H1-O8" one. This suggests that the former corresponds to the most stable bond when no cholesterol is present, but that is, it becomes weaker when the amount of cholesterol increases. Conversely, the HB between "H1" and "O8" becomes strongest at the membrane with $50 \%$ content of cholesterol. Then we can observe the influence of cholesterol in the TRP-DPPC binding as one major effect, probably related with the relative orientation between the duo. Concerning "H2"-DPPC binding (right column of Fig. 6), we observed its existence in all analyzed setups, although the maxima are found at slightly larger distances (around 1.9-2.0 ). In the same fashion as for "H1"-DPPC binding, the presence of cholesterol showed significant influence on the characteristics of "H2"-DPPC bonds as well. Finally, the structure of water around DPPC has been found to be very similar to that of water around DMPC, as was previously described in Ref. 47, Sec. III.

In order to estimate the averaged time intervals for TRPlipid association, we display the time evolution of selected atom-atom distances in Fig. 7. There we can see that typical hydrogen-bonding distances are reached in all cases. At the cholesterol-free membrane, one of the three hydrogens of tryptophan bound to its "N1" site is almost permanently bound to the oxygen "O2" of DPPC with some intervals of simultaneous binding ("shared" hydrogen-bonds). At the two cholesterol concentrations considered here, the binding is less common, with a tendency to longer hydrogen-bonding periods at the $30 \%$ concentration. In all cases, the typical H-bond lifetimes are of the order of $1 \mathrm{~ns}$, with eventual intervals of breaking and re-formation of hydrogen-bonds.

When analyzing the hydrogen-bonding of TRP with cholesterol, we have observed that some periods of hydrogenbonding are established between oxygen "O2" of TRP and the hydroxyl's hydrogen of cholesterol ("HC") and that, simultaneously, one of the TRP's hydrogens (bound to "N1") forms some HBs with hydroxyl's oxygen of cholesterol. Again we see longer binding for cholesterol's $30 \%$ concentration. The time scale of hydrogen-bonding seems shorter than $1 \mathrm{~ns}$, although more precise calculations of hydrogen-bond lifetimes using
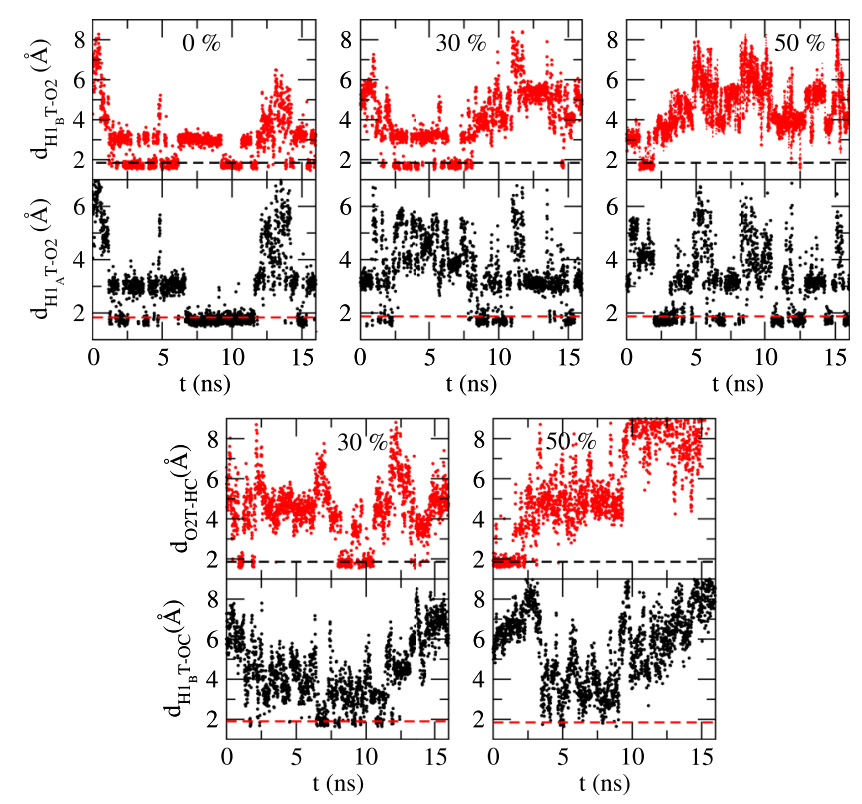

FIG. 7. Distance distribution of selected sites in cholesterol-TRP bonding and DPPC-TRP bonding as a function of simulation time. Percentages of cholesterol are indicated on top of each graph. Dashed lines indicate the typical HB distances obtained from Fig. $6(1.8 \AA)$. " $\mathrm{H} 1_{A} \mathrm{~T}$ " and " $\mathrm{H} 1_{B} \mathrm{~T}$ " are two of the three hydrogens bound to the site "N1" of tryptophan, whereas "O2" is one of two oxygens of TRP and "OC" ("HC") is the hydroxyl's oxygen (hydrogen) of cholesterol.

correlation functions (see Ref. 48 for instance) are out of the scope of this paper and have not been carried out.

In order to have a more detailed idea on TRP binding to the membrane, we are reporting two characteristic snapshots of TRP linked to DPPC and cholesterol simultaneously (see Fig. 8). There we can observe that either TRP can be bound to two DPPC in different sites ("O2" and "O8") and one cholesterol (right) or it can be bound to one DPPC (site "O2") and to two cholesterol molecules, the first through the hydroxyl's hydrogen and the second through hydroxyl's oxygen (left). These images help to enlighten the relatively complex multiple hydrogen-bonding connections between TRP, DPPC, and cholesterol described above (see Sec. III A) and the key role of TRP as a bridging molecule between several lipid or cholesterol chains.

\section{B. Dynamics: Diffusion and vibrational spectra}

\section{Diffusion of tryptophan and water}

Microscopic dynamics of tryptophan and water have been extensively considered. The first property evaluated has been the mean square displacement (MSD) of water (monitoring oxygen's trajectories) and of the carbon "C2" in TRP (see Fig. 1). Diffusion coefficients of DPPC require simulations at the scale of hundreds of nanoseconds and have not been computed here, although a detailed study on DMPC was reported earlier. ${ }^{47}$ From the long-time slopes of both MSD, we obtained the corresponding self-diffusion coefficients $D$ through Einstein's formula

$$
D=\lim _{t \rightarrow \infty} \frac{\left\langle\left|\vec{r}_{i}(t)-\vec{r}_{i}(0)\right|^{2}\right\rangle}{2 d \Delta t},
$$




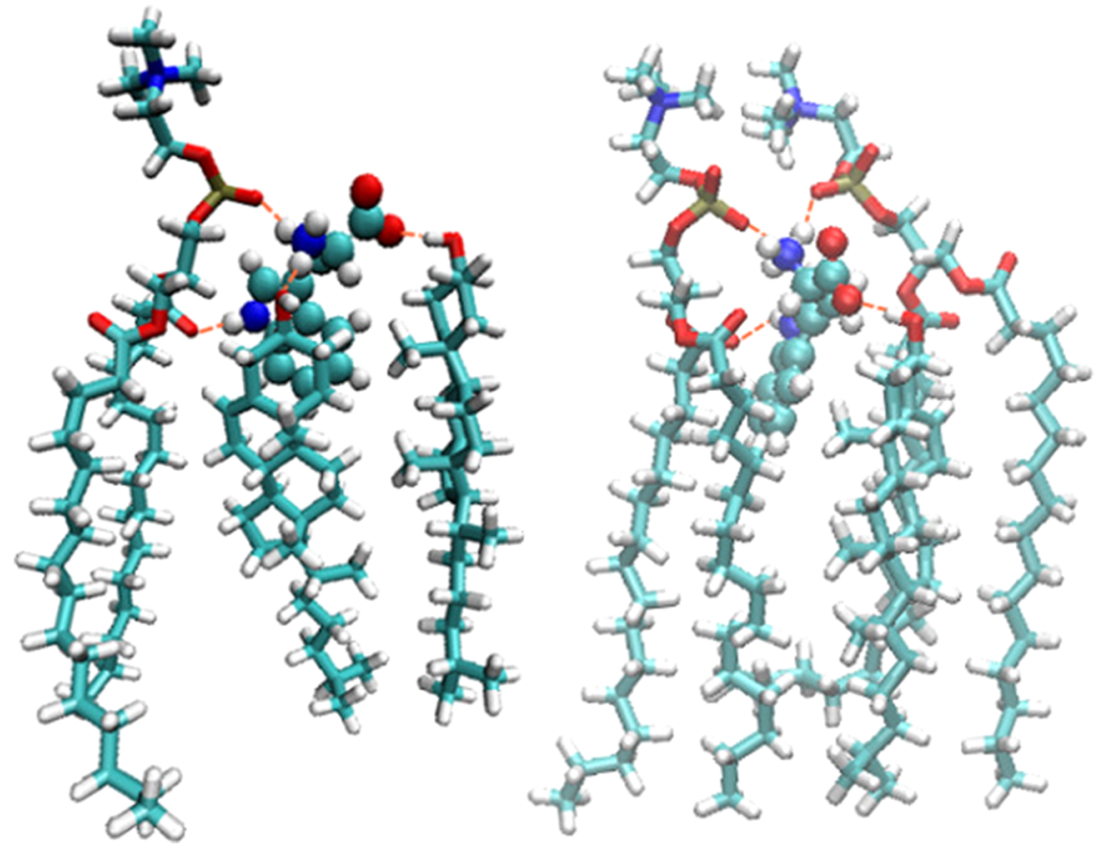

FIG. 8. Snapshots of typical DPPCtryptophan-cholesterol bonds. Atoms in TRP colored are as in Fig. 2. Binding sites have been highlighted in dashed orange lines. where $\vec{r}_{i}$ is the instantaneous position of particle $i$. In this general procedure, the spatial dimension of the diffusion regions $d$ is considered. So, water undertakes isotropical diffusion $(d=3)$ meanwhile tryptophan showed lateral-like diffusion $(d=2)$. The results have been summarized in Table II.

In the case of water, the results include all molecules in the system regardless of their location at bulk or interfacial regions. In a previous study, ${ }^{47}$ we obtained $D$ separately for bound water, water at interfaces, and water external to the membrane and found the mobility of water molecules being significantly faster as their distance to the interface increased (water outside the membrane was about 14 times faster than water bound to lipid chains). In this work, we simply included water's diffusion for the sake of comparison with those previous results in order to test the influence of TRP and of cholesterol. At $0 \%$ cholesterol, our results are of the same order of magnitude as the ones obtained for the simple aqueous DMPC membrane ${ }^{47}$ at $303 \mathrm{~K}\left(2.66 \times 10^{-5}\right)$ which indicates that, as expected, the presence of TRP does not affect significantly water's dynamics. Nevertheless, we can observe that when cholesterol is included in the system, water tends to diffuse about $33 \%$ faster. This can be attributed to the fact that at non-zero cholesterol concentrations, membranes are more compact (areas per lipid are smaller) and water's penetration in the interface is harder to occur, producing larger diffusion mainly along the surface of the bilayer.

TRP's diffusion coefficients were computed from simulation runs where TRP was permanently at the interface.

TABLE II. Self-diffusion coefficients $D\left(\mathrm{in}^{2} / \mathrm{s}\right)$ of water and of tryptophan as a function of the cholesterol's percentage of the membrane.

\begin{tabular}{lccc}
\hline \hline$D$ & $0 \%$ & $30 \%$ & $50 \%$ \\
\hline Water & $5.03(0.01) \times 10^{-5}$ & $4.20(0.01) \times 10^{-5}$ & $4.45(0.07) \times 10^{-5}$ \\
Tryptophan & $3.48(0.80) \times 10^{-7}$ & $2.91(0.35) \times 10^{-7}$ & $1.40(0.02) \times 10^{-6}$ \\
\hline \hline
\end{tabular}

We stress this point because in our simulation runs, we observed that TRP is able to make excursions to the water bulk regions, especially when cholesterol concentration increases. For instance, at the highest concentration of 50\%, TRP will stay eventually half of the time at the water bulk and the remaining time at the lipid-water interface. Along the periods when TRP was fully solvated by water, we estimated that its diffusion coefficient was $3.9 \times 10^{-6}$ at $50 \%$ concentration of cholesterol. The latter value is significantly higher than those corresponding to TRP at the lipid-water interfaces (see Table II).

Focusing on the diffusion of TRP when residing at the interface, the values reported in Table II are about two orders of magnitude smaller than those of water (for cholesterol concentration up to $30 \%$ ). At the highest concentration $(50 \%)$, the diffusion of TRP becomes significantly faster. The values of $D$ for TRP ( $0 \%$ cholesterol's concentration) are of the same order of magnitude of those of DMPC molecules ${ }^{47}$ $\left(0.6 \times 10^{-7}\right.$ in the absence of cholesterol), although the former are about 6 times smaller. This indicates that TRP's mobility is significantly higher than that of DPPC, and this suggests that the mechanisms of diffusion of TRP would be qualitatively different of those of lipids (suggested to be due to the motion of nanodomains or local groups of around 10 units 47 and also directly related with the slow motion of gel-like tails in the present case). We should point out that the effect of temperature is remarkable since at complementary simulations at $310 \mathrm{~K}$, TRP diffusion was of about $2 \times 10^{-7}$, i.e., significantly slower, due to the essentially gel-like state of the membrane at such conditions.

Regarding the reliability of the present calculations, we should indicate that the values reported in Table II have been obtained in the framework of the CHARMM36 force field. Whereas the number of lipids in our systems may be large enough $^{49}$ to avoid strong size effects from periodic boundary conditions, there are some known deficiencies of the 
CHARMM36 (and the MARTINI force field among others) regarding its reliability, to be considered when computing lipid diffusion ${ }^{50}$ to be considered. Furthermore, Yeh and Hummer suggested that, in simulations using periodic boundary conditions, hydrodynamic interactions should be taken into account in order to obtain more accurate values of $D$, in such a way that a correction of the order $N^{\frac{1}{3}}$ might be in order. ${ }^{51}$ In our case, we did not consider lipid diffusion but tryptophan's. Since tryptophan dynamics are clearly faster than DPPC's, we expect that our computed $D$ are correct in order of magnitude, although they might presumably be affected at some extent by the deficiencies of the force field mentioned above. Only the existence of precise experimental values could shed light in this issue, but up to our best knowledge, no measured diffusion coefficients of tryptophan in zwitterionic membranes are currently available.

\section{Spectral densities of tryptophan}

We have computed and analyzed the spectral densities of the tryptophan molecule. Experimental infrared spectra are usually obtained through the absorption coefficient $\alpha(\omega)$ or the imaginary part of the frequency-dependent dielectric constant. ${ }^{52}$ These properties are directly related to the absorption line shape $I(\omega)$, which can be obtained from molecular dynamics simulations ${ }^{53-55}$ in certain cases. In most cases, the physically relevant property to be computed is the so-called atomic spectral density $S_{i}(\omega)$, defined as

$$
S_{i}(\omega)=\int_{0}^{\infty} \mathrm{d} t\left\langle\vec{v}_{i}(t) \vec{v}_{i}(0)\right\rangle \cos (\omega t)
$$

where $\vec{v}_{i}(t)$ is the velocity of atom $i$. In our case, we have obtained the spectral density of the whole tryptophan molecule as the sum of the contributions of all atoms pertaining to the molecule. In formula (4), the brackets $\langle\cdots\rangle$ denote equilibrium ensemble average. Generally speaking, classical molecular dynamics simulations are not able to fully reproduce experimental absorption coefficients, these being quantum properties. However they can be used to locate the position of the spectral bands since in the harmonic (oscillator) approximation, classical and quantum fundamental frequencies are the same. The model employed in the present work considers harmonic bond vibrations, and for such a reason, we will be able to locate the positions of the main experimental spectral bands. We are reporting the full spectral density $S_{T R P}(\omega)$ in the left side of Fig. 9.

Our results reveal two main spectral regions, one for frequencies below $1800 \mathrm{~cm}^{-1}$ and another one for $2700<\omega$ $<3400 \mathrm{~cm}^{-1}$. The agreement with available data from infrared and Raman spectroscopy of tryptophan in aqueous solution reported by Leyton et al. $^{7}$ (and references therein) is very good overall. These authors reported strong maxima located at 128 , 163, 425 (weak), 744, 1014, (1351), 1432, 1566, (1647), 2938, 3056 , and $3402 \mathrm{~cm}^{-1}$. The numbers into parentheses indicate a group of maxima centered at the reference value indicated here. Most of these maxima are included in the $S(\omega)$ reported in Fig. 9 and, at $0 \%$ cholesterol content, show excellent agreement with only three clear disagreements: (1) the Raman peak
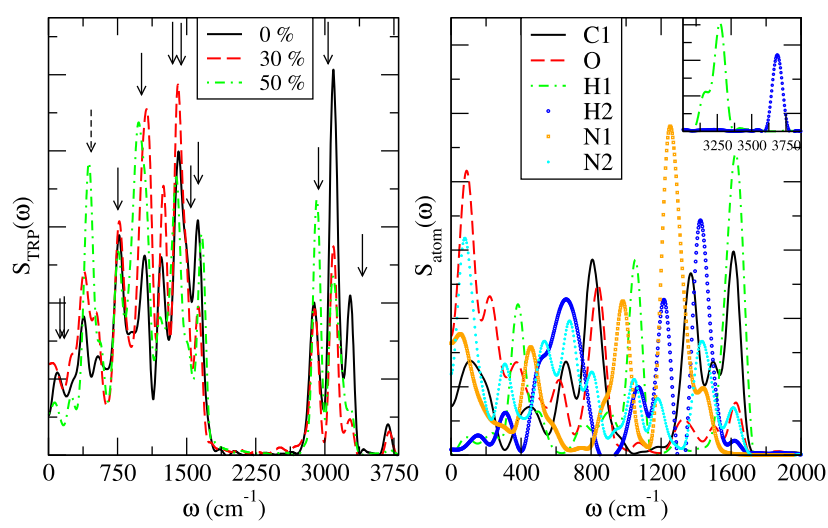

FIG. 9. Spectral densities of the full tryptophan molecule (left). Full arrows indicate the position of experimental peaks reported in Ref. 7. The dashed arrow indicates a maximum of weak intensity. Decomposition of the spectrum for the $0 \%$ cholesterol case (right), indicating the relative contribution of several (most relevant) selected atoms. The inset reveals the contributions of high frequencies of the two relevant groups of hydrogen atoms ("H1" and "H2").

located at $163 \mathrm{~cm}^{-1}$ is not clearly seen in $S_{T R P}(\omega)$; (2) the same fact happens with the band located at $1566 \mathrm{~cm}^{-1}$ (unseen), and (3) the Raman peak at the highest frequency at $3402 \mathrm{~cm}^{-1}$ is strongly red-shifted in the computed spectral density (reported at $3276 \mathrm{~cm}^{-1}$ ).

Having established the reliability of the potential model and method employed in the present work, we have obtained the partial spectra of each atom in TRP. The results are reported in the right side of Fig. 9. There we gain advantage of one of strongest points of molecular dynamics simulations: we can discern the contribution of each atom to the full spectrum. We should remember that frequencies below $1000 \mathrm{~cm}^{-1}$ are usually related to rotational and librational motions, those between 1000 and $1500 \mathrm{~cm}^{-1}$ are normally attributed to bending motions, and those frequencies of the order of 2000-3000 wavenumbers are due to stretching motions (for liquid water, see, for instance Ref. 56). The list of assignments of this work is as follows:

1. The peak experimentally found at $128 \mathrm{~cm}^{-1}$ corresponds, within our framework, to restricted translations of oxygens since it corresponds to the high maximum (centered at $123 \mathrm{~cm}^{-1}$ ) appearing at the oxygen's spectral density. Furthermore, it can also be related with carbon vibrations (maxima at 95 and $210 \mathrm{~cm}^{-1}$ ). In summary, we can attribute this band to " $\mathrm{C} 1-\mathrm{O}$ " vibrations (see Fig. 1).

2. The peak located at $163 \mathrm{~cm}^{-1}$ is not seen in our spectral decomposition.

3. The weak band centered around $425 \mathrm{~cm}^{-1}$ is matched by the maxima at $385 \mathrm{~cm}^{-1}$ of our spectrum. This indicates that this vibration should be attributed to a low-frequency vibration of hydrogens "H1" (see Fig. 1). Since this is a relatively low frequency, it will probably correspond to a rotational motion.

4. The maximum at $744 \mathrm{~cm}^{-1}$ is located around $810 \mathrm{~cm}^{-1}$ in Fig. 9, and it appears in both the contributions of atoms, carbon "C1" and oxygens "O" (TRP has two of them), suggesting a "scissoring" motion of the corresponding triplet. 
5. The peak at $1014 \mathrm{~cm}^{-1}$ is found at 1045 in the present work, and it is directly related with the spectra of sites "N1" and "H1," suggesting, in this case, a bond stretch of those particles.

6. The group of experimental bands centered around $1351 \mathrm{~cm}^{-1}$ is matched in our spectra by one peak centered at $1225 \mathrm{~cm}^{-1}$. Connected to particles "N1" and one carbon, we expect that this band should correspond to the stretch of "N1" along its carbon bond.

7. The maximum at $1432 \mathrm{~cm}^{-1}$ can be directly related with the maxima around $1410 \mathrm{~cm}^{-1}$ observed in the spectra of "N2" and "H2," suggesting that this vibration corresponds to a bond stretch between them.

8. The next band, centered at $1566 \mathrm{~cm}^{-1}$, is not well matched by any peak of the computed spectrum.

9. The remaining band centered at $1647 \mathrm{~cm}^{-1}$ is a group of maxima in the experimental spectrum, and it can be also attributed to the contribution of "H1" $\left(1622 \mathrm{~cm}^{-1}\right)$. Since the label "H1" accounts for the three hydrogens bound to "N1," it should be attributed to a single stretching mode.

10. Finally, the three bands of the highest frequency (2938, 3056 , and $3402 \mathrm{~cm}^{-1}$ ) must be fully attributed to stretching vibrations of hydrogens "H1"and "H2" and are located around 3170 (shoulder), 3270, and $3690 \mathrm{~cm}^{-1}$ in our spectral densities. The large deviations not only indicate a drawback in the characterizations of the vibrational interactions by the force field employed here but they also indicate their origin based on pure hydrogen vibrations.

The influence of cholesterol in the full spectrum of TRP reported in the left side of Fig. 9 is remarkable in some of the spectral bands, producing observable frequency shifts, and it essentially indicates again that cholesterol interacts with TRP in a quite strong way and that these interactions are able to modify the vibrational motions of their atomic components producing frequency shifts up to $66 \mathrm{~cm}^{-1}$ (the case of the band centered around $1045 \mathrm{~cm}^{-1}$ in the computed spectrum, red-shifted at the system with $50 \%$ of cholesterol).

\section{CONCLUDING REMARKS}

A series of molecular dynamics simulations of a DPPC lipid bilayer membrane in an aqueous ionic solution of $\mathrm{NaCl}$ with an embedded single tryptophan molecule and at three cholesterol concentrations $(0 \%, 30 \%$ and $50 \%)$ have been performed by MD using the CHARMM36 force field. We have first focused our analysis on the local structure of zwitterion L-tryptophan, when associated with water, DPPC, and cholesterol molecules. After the systematic analysis of meaningful data, we noted relevant changes in the local structure and dynamics of TRP only for cholesterol concentrations above $30 \%$. In agreement with MacCallum et al. ${ }^{46}$ we observed that TRP tends to localize strongly in the interfacial region so that crossing the membrane by diffusion with no help of external fields or mediating particles is unlikely to happen in the time scale of our simulations. The radial distribution functions were evaluated for six classes of particles: the hydrogens labeled (1) "H1," (2) "H2," (3) the double bonded oxygens "O1" and "O2" of tryptophan, (4) the three water sites, (5) the charged oxygens labeled "O2" and "O8" of DPPC, and (6) the oxygen and hydrogen sites of the hydroxyl group of cholesterol.

Our data revealed the existence of a strong first coordination shell and a milder second coordination shell for the TRP-water structure. The binding of tryptophan to DPPC involved coordination shells for the different sites of possible association [charged oxygens "O2" and "O8" of DPPC versus the two tagged hydrogens in TRP ("H1" and "H2")]. Finally, the distribution functions of TRP-cholesterol revealed stable hydrogen-bonding configurations. These data indicate that TRP is able to establish stable interactions with all classes of solvating particles (water, DPPC, and cholesterol) including a sort of bridging between DPPC and cholesterol species. The typical hydrogen-bond distances between TRP and other species have been found between 1.7 and $2.0 \AA$, in good agreement with experimental data obtained from fluorescence measurements. ${ }^{8}$

Tryptophan self-diffusion coefficients have been found to be of the order of $1-10 \times 10^{-7} \mathrm{~cm}^{2} / \mathrm{s}$, and they are strongly depending of the presence of cholesterol in the system. The diffusion of TRP has revealed to be significantly faster than that of DPPC and slower than that of water. The computed spectral densities, in excellent agreement with experimental infrared and Raman data from Leyton et al., ${ }^{7}$ allowed us to reveal the degree of participation of each atomic site of tryptophan to the complete spectrum of the molecule.

\section{ACKNOWLEDGMENTS}

The authors gratefully acknowledge financial support provided by the Spanish Ministry of Economy and Knowledge (Grant No. FIS2015-66879-C2-1-P). H.L. is the recipient of a grant from the Chinese Scholarship Council (No. 201607040059). Computational resources from the Barcelona Supercomputing Center-Spanish Supercomputing Network (Grant Nos. FI-2018-1-0007 and FI-2018-2-0018) are also acknowledged.

${ }^{1}$ O. G. Mouritsen, Life-As a Matter of Fat (Springer, 2005).

${ }^{2}$ J. F. Nagle and S. Tristram-Nagle, Biochim. Biophys. Acta, Biomembr. 1469, 159 (2000).

${ }^{3}$ H. Ohvo-Rekilä, B. Ramstedt, P. Leppimäki, and J. P. Slotte, Prog. Lipid Res. 41, 66 (2002).

${ }^{4}$ T. P. McMullen, R. N. Lewis, and R. N. McElhaney, Curr. Opin. Colloid Interface Sci. 8, 459 (2004).

${ }^{5}$ H. I. Petrache, S. W. Dodd, and M. F. Brown, Biophys. J. 79, 3172 (2000).

${ }^{6}$ K. M. Sanchez, G. Kang, B. Wu, and J. E. Kim, Biophys. J. 100, 2121 (2011).

${ }^{7}$ P. Leyton, J. Brunet, V. Silva, C. Paipa, M. V. Castillo, and S. A. Brandán, Spectrochim. Acta, Part A 88, 162 (2012).

${ }^{8}$ H. Liu, H. Zhang, and B. Jin, Spectrochim. Acta, Part A 106, 54 (2013).

${ }^{9}$ M. A. Digman, V. R. Caiolfa, M. Zamai, and E. Gratton, Biophys. J. 94, L14 (2008).

${ }^{10}$ L. Malacrida, D. M. Jameson, and E. Gratton, Sci. Rep. 7, 9215 (2017).

${ }^{11}$ S. Schurch, M. Lee, and P. Gehr, Pure Appl. Chem. 64, 1745 (1992).

${ }^{12}$ G. S. Ayton and G. A. Voth, Biophys. J. 87, 3299 (2004).

${ }^{13}$ A. Slominski, I. Semak, A. Pisarchik, T. Sweatman, A. Szczesniewski, and J. Wortsman, FEBS Lett. 511, 102 (2002).

${ }^{14}$ A. J. de Jesus and T. W. Allen, Biochim. Biophys. Acta, Biomembr. 1828, 864 (2013).

${ }^{15}$ I. Kostoglou-Athanassiou, Ther. Adv. Endocrinol. Metab. 4, 13 (2013).

${ }^{16}$ J. Guo, Q. Xu, Z. Zheng, S. Zhou, H. Mao, B. Wang, and F. Yan, ACS Macro Lett. 4, 1094 (2015). 
${ }^{17}$ F. Shima, T. Akagi, and M. Akashi, Colloid Polym. Sci. 292, 2663 (2014).

${ }^{18}$ S. Jo, T. Kim, V. G. Iyer, and W. Im, J. Comput. Chem. 29, 1859 (2008).

${ }^{19}$ S. Jo, J. B. Lim, J. B. Klauda, and W. Im, Biophys. J. 97, 50 (2009).

${ }^{20}$ W. L. Jorgensen, J. Chandrasekhar, J. D. Madura, R. W. Impey, and M. L. Klein, J. Chem. Phys. 79, 926 (1983).

${ }^{21}$ J. L. Thewalt and M. Bloom, Biophys. J. 63, 1176 (1992).

${ }^{22}$ N. Kučerka, M.-P. Nieh, and J. Katsaras, Biochim. Biophys. Acta, Biomembr. 1808, 2761 (2011).

${ }^{23}$ J. C. Phillips, R. Braun, W. Wang, J. Gumbart, E. Tajkhorshid, E. Villa, C. Chipot, R. D. Skeel, L. Kalé, and K. Schulten, J. Comput. Chem. 26, 1781 (2005).

${ }^{24}$ H. J. Berendsen, J. V. Postma, W. F. van Gunsteren, A. DiNola, and J. Haak, J. Chem. Phys. 81, 3684 (1984).

${ }^{25}$ S. E. Feller, Y. Zhang, R. W. Pastor, and B. R. Brooks, J. Chem. Phys. 103, 4613 (1995).

${ }^{26}$ J. B. Klauda, R. M. Venable, J. A. Freites, J. W. O'Connor, D. J. Tobias, C. Mondragon-Ramirez, I. Vorobyov, A. D. MacKerell, and R. W. Pastor, J. Phys. Chem. B 114, 7830 (2010).

${ }^{27}$ J. B. Lim, B. Rogaski, and J. B. Klauda, J. Phys. Chem. B 116, 203 (2012).

${ }^{28}$ U. Essmann, L. Perera, M. L. Berkowitz, T. Darden, H. Lee, and L. G. Pedersen, J. Chem. Phys. 103, 8577 (1995).

${ }^{29}$ J. Martí Rabassa and H. Lu, Procedia Comput. Sci. 108, 1242 (2017).

${ }^{30}$ G. W. Stockton and I. C. Smith, Chem. Phys. Lipids 17, 251 (1976).

${ }^{31}$ C. Hofsäß, E. Lindahl, and O. Edholm, Biophys. J. 84, 2192 (2003).

${ }^{32}$ T. P. Trouard, A. A. Nevzorov, T. M. Alam, C. Job, J. Zajicek, and M. F. Brown, J. Chem. Phys. 110, 8802 (1999).

${ }^{33}$ O. Berger, O. Edholm, and F. Jähnig, Biophys. J. 72, 2002 (1997).

${ }^{34}$ S. Chiu, E. Jakobsson, R. J. Mashl, and H. L. Scott, Biophys. J. 83, 1842 (2002).

${ }^{35}$ P. R. Pandey and S. Roy, J. Phys. Chem. B 115, 3155 (2011).

${ }^{36}$ O. Edholm and J. F. Nagle, Biophys. J. 89, 1827 (2005).
${ }^{37}$ Y. Wang, P. Gkeka, J. E. Fuchs, K. R. Liedl, and Z. Cournia, Biochim. Biophys. Acta, Biomembr. 1858, 2846 (2016).

${ }^{38}$ C. L. Armstrong, M. A. Barrett, A. Hiess, T. Salditt, J. Katsaras, A.-C. Shi, and M. C. Rheinstädter, Eur. Biophys. J. 41, 901 (2012).

39 J. Yang, J. Martí, and C. Calero, Soft Matter 12, 4557 (2016).

${ }^{40} \mathrm{D}$. Chandler, Introduction to Modern Statistical Mechanics (Oxford University Press, 1987).

${ }^{41}$ J. Martí, F. S. Csajka, and D. Chandler, Chem. Phys. Lett. 328, 169 (2000).

${ }^{42}$ P. G. Bolhuis, D. Chandler, C. Dellago, and P. L. Geissler, Annu. Rev. Phys. Chem. 53, 291 (2002).

${ }^{43}$ J. Martí and F. S. Csajka, Phys. Rev. E 69, 061918 (2004).

${ }^{44}$ A. Barducci, G. Bussi, and M. Parrinello, Phys. Rev. Lett. 100, 020603 (2008).

${ }^{45}$ J. Yang, C. Calero, M. Bonomi, and J. Martí, J. Chem. Theor. Comput. 11, 4495 (2015).

${ }^{46}$ J. L. MacCallum, W. D. Bennett, and D. P. Tieleman, Biophys. J. 94, 3393 (2008).

${ }^{47}$ J. Yang, C. Calero, and J. Martí, J. Chem. Phys. 140, 104901 (2014).

${ }^{48}$ J. Marti, Phys. Rev. E 61, 449 (2000).

${ }^{49}$ J. B. Klauda, B. R. Brooks, and R. W. Pastor, J. Chem. Phys. 125, 144710 (2006).

${ }^{50}$ R. M. Venable, H. I. Ingólfsson, M. G. Lerner, B. S. Perrin, Jr., B. A. Camley, S. J. Marrink, F. L. Brown, and R. W. Pastor, J. Phys. Chem. B 121, 3443 (2017).

${ }^{51}$ I.-C. Yeh and G. Hummer, J. Phys. Chem. B 108, 15873 (2004).

${ }^{52}$ D. A. McQuarrie, Statistical Mechanics (University Science Books, 2000).

${ }^{53}$ J. Martí, J. Padró, and E. Guardia, Mol. Simul. 11, 321 (1993).

${ }^{54}$ J. Martí, E. Guardia, and J. Padró, J. Chem. Phys. 101, 10883 (1994).

${ }^{55}$ M. Praprotnik and D. Janežič, J. Chem. Phys. 122, 174103 (2005).

${ }^{56} \mathrm{D}$. Eisenberg and W. Kauzmann, The Structure and Properties of Water (Oxford University Press, 2005). 